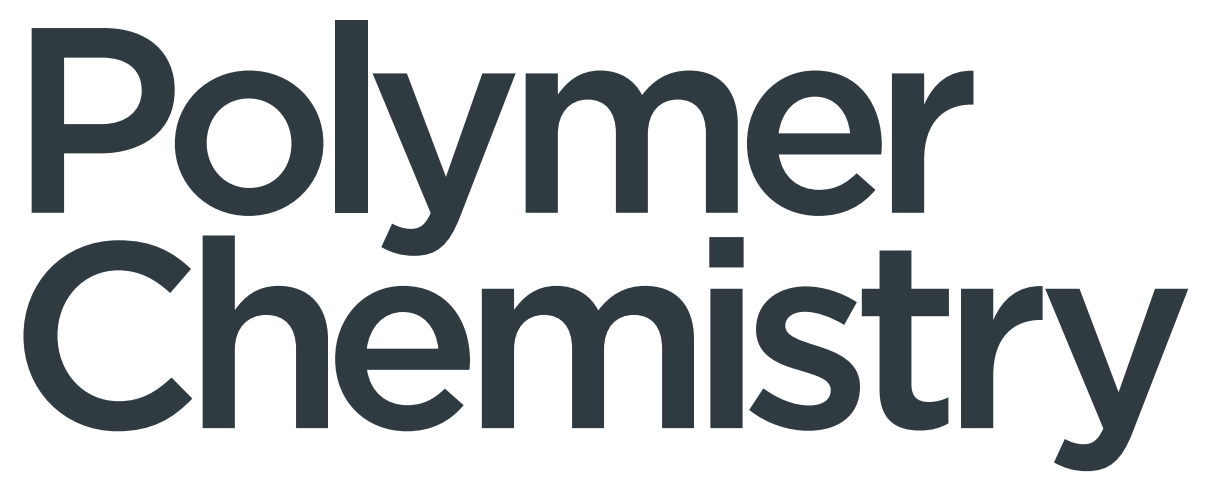

Volume 11

Number 25

7 July 2020

Pages 4061-4194

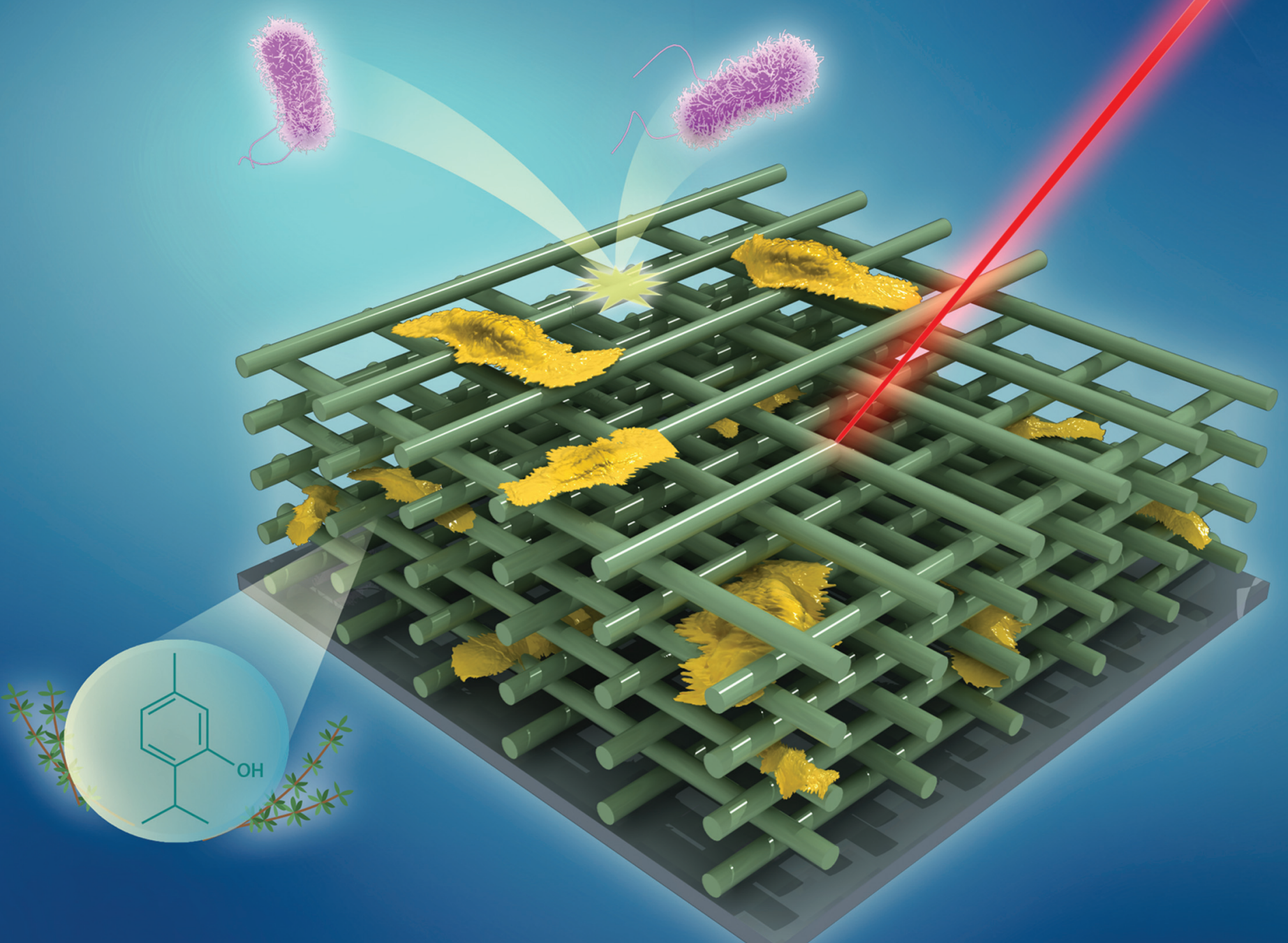

ISSN 1759-9962

\title{
COMMUNICATION
}

Maria Vamvakaki et al.

ROYAL SOCIETY

Multi-photon polymerization of bio-inspired, thymol-

functionalized hybrid materials with biocompatible and antimicrobial activity 
D) Check for updates

Cite this: Polym. Chem., 2020, 11, 4078

Received 20th February 2020,

Accepted 18th April 2020

DOI: 10.1039/d0py00281j

rsc.li/polymers

\section{Multi-photon polymerization of bio-inspired, thymol-functionalized hybrid materials with biocompatible and antimicrobial activity $\dagger$}

\author{
Kostas Parkatzidis, (iD a,b Maria Chatzinikolaidou, (iD a,c Eleftherios Koufakis, a,c \\ Maria Kaliva, ${ }^{a, c}$ Maria Farsari ${ }^{a}$ and Maria Vamvakaki (iD *a,c
}

\begin{abstract}
Multi-functional materials are of paramount importance in biomedical engineering. In this work, we present the synthesis of a hybrid, organic-inorganic material functionalized with covalentlyattached natural antimicrobial moeties based on thymol, an essential oil found in thyme. The hybrid material is a double organic-inorganic network formed by sol-gel chemistry followed by photopolymerizaton of the organic moeities, and allows the fabrication of highly accurate 3D scaffolds by means of multi-photon polymerization. The cytocompatibility of the materials and scaffolds was proved by the excellent cell adhesion and proliferation of dental pulp stem cells, whereas their osteogenic potential was evidenced by the significant production of calcium in the matrix, following functionalization with bone morphogenetic protein-2. The antimicrobial activity of the thymol-functionalized 3D scaffolds against $E$. coli and $B$. cereus was demonstrated by the inhibition of bacterial growth after 1 and 4 days in culture. These dual-functional, hybrid materials, exhibiting simultaneously biocompatibility and osteogenic potential, as well as an effective antimicrobial activity are excellent candidates for bone tisue engineering.
\end{abstract}

Multi-functional materials have drawn great attention over the last years. ${ }^{1-3}$ The unique combination of diverse material properties and the material response to a variety of external stimuli are of paramount importance in many research fields, among which drug delivery and tissue engineering are the most prominent ones. ${ }^{4}$ Smart polymeric biomaterials should closely mimic the tissue and/or organs they will regenerate or support, while simultaneously respond to the dynamic

\footnotetext{
${ }^{a}$ Institute of Electronic Structure and Laser, Foundation for Research and TechnologyHellas, Heraklion, Greece. E-mail:vamvakak@iesl.forth.gr

${ }^{b}$ Department of Chemistry, University of Crete, Heraklion, Greece

${ }^{c}$ Department of Materials Science and Technology, University of Crete, Heraklion, Greece

$\dagger$ Electronic supplementary information (ESI) available: Experimental details, synthetic and scaffold fabrication procedures, material characterization, cell and bacteria culture assays, optical microscopy and SEM images of cells and bacteria. See DOI: $10.1039 / \mathrm{d} 0$ py00281j
}

changes of the biological environment., ${ }^{5,6}$ Major challenges encountered in the implantation of biomaterials nowadays are their rejection by the body, due to the lack of biocompatibility, and the postoperative infection of the constructs, which requires revision surgery. Therefore, the synthesis of biomaterials that combine good biocompatibility with excellent antimicrobial properties is urgently needed. Polymeric, ${ }^{7,8}$ inorganic $^{9}$ as well as hybrid, organic-inorganic, materials have been shown to exhibit good biocompatibility, ${ }^{10}$ which is combined with an effective antimicrobial action conferred by the incorporation of active antimicrobial species, such as silver ${ }^{11}$ and zinc metals ${ }^{12}$ or organic compounds, i.e. chlorhexidine, ${ }^{13}$ quaternary ammonium salts, ${ }^{14}$ antimicrobial peptides, ${ }^{15}$ amine containing polymers, ${ }^{16,17}$ etc. However, in the majority of these cases, the gradual release of the antibacterial compounds from the constructs results in a short-term antibacterial action and toxic side effects to the surrounding tissues, whereas the functionalization of the material with the antimicrobial species can have a detrimental effect on its biocompatibility, impairing the implant integration in the body. ${ }^{18,19}$

An important class of antimicrobial agents are natural products $^{20}$ which exhibit remarkable biological properties through their high activity and selectivity. Recently, many biological and engineering studies have suggested the use of natural products as novel compounds for the control of biological events and disease, ${ }^{21}$ however, the vast majority of these studies explores the release of these compounds from different types of materials, leading to short-term antimicrobial activity and high cell toxicity. The development of biocompatible materials bearing immobilized antimicrobial moieties has been proposed to overcome the above challenges. While these materials inhibit the activity of contacted bacteria, the active agents are chemically bound to the matrix and cannot leach out. 2-Isopropyl-5-methylphenol (thymol), an essential oil extracted from Thymus vulgaris (thyme), is extensively used in dentistry to treat oral infections, due to its strong bacteriostatic and bactericidal activity against a wide range of bacteria. ${ }^{21,22}$ However, to the best of our knowledge, 
there are only three reports on the synthesis of polymeric materials bearing chemically-bound thymol moieties. Moszner et al. has described the synthesis and free-radical polymerization of thymyl methacrylate (THYMA) and $p$-styrene sulfonate. ${ }^{23}$ The antibacterial activity of poly(thymyl methacrylate) in a water suspension against Streptococcus mutans was attributed to the enzymatic release of thymol upon ester hydrolysis. In another study, Prudencio et al. reported the synthesis of antiseptic polyanhydrides bearing pendant thymyl groups. ${ }^{24}$ Although, the cell compatibility was shown at low polymer concentrations for L929 mouse fibroblast cells, the antimicrobial action of the polymers was not studied. More recently, Bedel and co-workers have reported the synthesis and antimicrobial activity of poly(thymyl methacrylate)-grafted poly(ethylene terephthalate) films. ${ }^{25}$ The antimicrobial studies showed that the polymer surface was highly resistant to bio-adhesion toward $P$. aeruginosa, L. monocytogenes, and $S$. aureus and strongly inhibited biofilm formation against $S$. aureus. However, none of these studies have investigated the dual-function, biocompatibility and antimicrobial activity, of the synthesized polymers, which is important for their application in the biomedical field.

Three-dimensional (3D) scaffolds have emerged as an attractive approach in tissue engineering to mimic more closely the cell environment and tissue regeneration processes in native tissues. ${ }^{26}$ Photo-bioprinting enables the fabrication of accurate 3D structures for use in tissue engineering and other biomedical applications, as well as in microoptics, nanophotonics, micro-/nanomechanics, and micro-nanofluidics. Among all bioprinting techniques, multiphoton lithography (MPL), based on multi-photon polymerization (MPP), has certain advantages over traditional extrusion or inkjet-based bioprinting. MPP is based on the localized polymerization/ cross-linking of photosensitive materials induced by femtosecond laser pulses. ${ }^{27}$ In MPP, the beam of an ultrafast NIR laser is tightly focused into the volume of a photosensitive material. In this very specific focal volume, a free-radical photopolymerization/photo-cross-linking process is initiated by the nonlinear absorption of photons from the photoinitiator. ${ }^{28}$ During the polymerization, the soluble monomers/macromers are converted into a solid polymer network insoluble in the solvent. By moving the beam in the three dimensions within the volume of the photosensitive material, arbitrary, highresolution 3D structures can be written. Finally, by simply immersing the sample in an appropriate solvent, the unscanned, unpolymerized area can be dissolved, revealing the $3 \mathrm{D}$ structures. ${ }^{29}$

In the present study, we synthesized a hybrid, organic-inorganic, material with covalently attached THYMA moieties. MPP was employed to fabricate accurate 3D porous scaffolds from this material, which were used in dental pulp stem cell (DPSC) and bacterial cultures for the assessment of its biocompatibility and antimicrobial activity, respectively. In addition to the dental cell adhesion and proliferation assessment on the thymol-functionalized hybrid material, we evaluated the osteogenic potential of the 3D scaffolds, upon functionalization with bone morphogenetic protein-2 (BMP-2), by quantifying the production of calcium in the extracellular matrix, and their antimicrobial action against Gram-negative and Gram-positive bacteria strains.

The hybrid material was synthesized by a sol-gel reaction of organically-modified metal alkoxide precursor compounds, followed by the photopolymerization of the organic groups to obtain the double network structure. In the first step, 3-(trimethoxysilyl)propyl methacrylate (MAPTMS) was hydrolyzed by $\mathrm{HCl}$ to yield hydroxyl silane groups and methanol as a byproduct of the reaction. In the second step, a mixture of 2-(dimethylamino)ethyl methacrylate (DMAEMA) and zirconium $n$-propoxide (ZPO) was added in the solution of hydrolyzed MAPTMS, and condensation between MAPTMS and ZPO took place to form the $\mathrm{Zr}-\mathrm{Si}$ oxide inorganic network. As reported in an earlier study from our group, DMAEMA acts simultaneously as a monomer and a scavenger in the MPP process, due to its methacrylate and amino functionalities, respectively, allowing to increase the resolution of the 3D printed structures without compromising their mechanical stability. ${ }^{30}$ To confer antimicrobial properties on the hybrid material, thymyl methacrylate, synthesized via the esterification of thymol with methacrylic anhydride (Scheme S1 and Fig. S1, S2 $\dagger$ ), was also added in the mixture. ${ }^{31}$ 4,4-Bis(diethylamino) benzophenone, was used as the photoinitiator to produce the radical species that will initiate the polymerization process. The above procedure produced a viscous liquid, which was drop-casted onto glass slides. The samples were allowed to dry in a vacuum oven to evaporate the methanol and propanol produced during the hydrolysis reactions, as well as the isopropanol of the ZPO solution. Once the samples were dry and condensation was complete, the drops were transformed into a gel which was photopolymerized (Fig. 1a). 3D microstructures were fabricated via MPP using an ultrafast pulsed laser at $780 \mathrm{~nm}$. A gridshaped porous scaffold with pore size $80 \mu \mathrm{m}$ and dimensions $555 \times 555 \times 50 \mu^{3}$ was designed for this study. This pore size was selected based on previous reports indicating a strong cell infiltration within the scaffold pores of $70-120 \mu \mathrm{m}$, followed by enhanced osteogenic and odontogenic differentiation ${ }^{32-34}$ The velocity $v s$. energy scanning of the laser allowed to determine the optimum fabrication parameters, which were $60 \mathrm{~mW}$ laser power and $1000 \mu \mathrm{m} \mathrm{s}^{-1}$ writing speed. This velocity-energy combination led to the formation of highly accurate 3D structures in 10 min. Fig. 1b-d shows the SEM images of the fabricated 3D scaffolds. The top view (Fig. 1b), as well as the $45^{\circ}$ tilted side view (Fig. 1c and d) images show highly accurate 3D porous scaffolds, with respect to the design dimensions. The produced 3D scaffolds confirm that the synthesized THYMAhybrid material is suitable for MPP and can be used for the fabrication of 3D structures. Next, DPSCs were cultured on the material films and 3D scaffolds. Representative optical microscopy images show the morphology of DPSCs cultured on the hybrid and THYMA-hybrid material films and on the polystyrene control surface for 2 and 7 days in culture (Fig. S3†). Cells seeded for 2 days in culture indicated a similar spindle-shaped morphology on the two film surfaces, the 

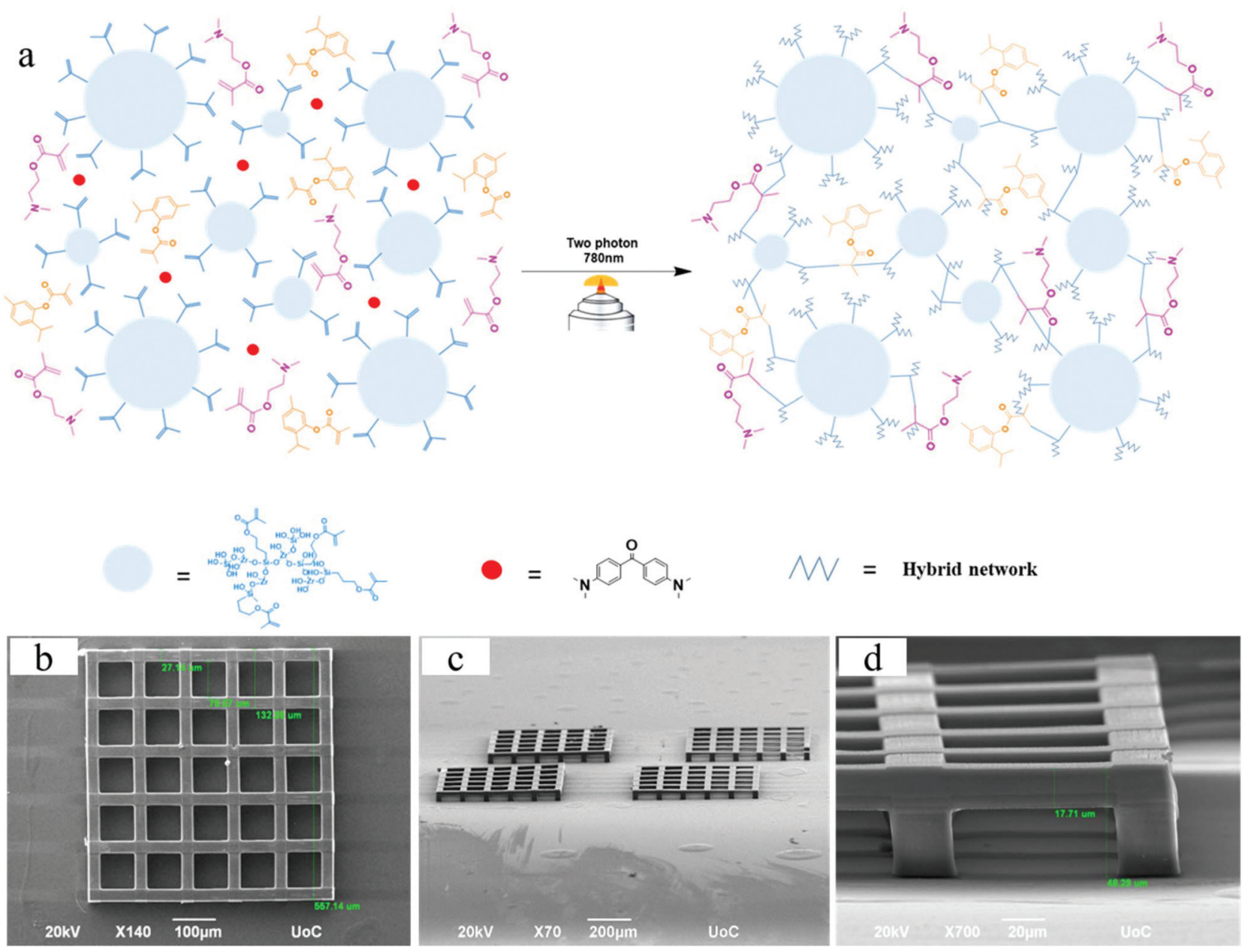

Fig. 1 (a) Schematic representation of the THYMA-hybrid material photopolymerization. 3D grid-shaped scaffolds produced by MPP, (b) top view, (c) side view and (d) side view at higher magnification, using a $20 \times$ plan achromat lens (N.A. $=0.8$ ) at 60 mW laser power and writing speed $1000 \mu \mathrm{m} \mathrm{s}^{-1}$.

hybrid and THYMA-hybrid material, which is similar to that on the polystyrene control surface (Fig. S3, $\uparrow$ top panel). After 7 days in culture, a clear increase in proliferation was observed with a dense layer of well-spread, flattened cells completely covering the material surface, as shown in the bottom panel of Fig. S3. $\dagger$ Fig. 2a shows the viability of the DPSCs on the hybrid and THYMA-hybrid material films assessed using the PrestoBlue ${ }^{\circledR}$ assay after 2, 4, and 7 days in culture. The cell viability was $\sim 100 \%$, compared to the tissue culture treated polystyrene control surface, for both materials at the three investigated time points. The material cytocompatibility was examined on geometrically well-defined films, in order to quantify the cell proliferation results. For both materials, an excellent initial cell adhesion and a subsequent cell proliferation increase, after 4 and 7 days in culture, was found. The cell number on both films increased approximately by two-fold from day 2 to day 4 , and 4 times from day 2 to day 7 . These results indicate the absence of any cytotoxic effects and suggest that the THYMAhybrid material is cytocompatible, and therefore, can be used for the fabrication of biomaterial scaffolds.
After examining the materials in 2D cell culture, in which they exhibited an excellent cell viability and proliferation profile, 3D porous scaffolds, made from the hybrid and THYMA-hybrid materials, were used to study the adhesion of the cells and bacteria in $3 \mathrm{D}$ culture. For this purpose, $2 \times 10^{4}$ DPSCs were cultured on a glass substrate with dimensions $1 \times$ $1 \mathrm{~cm}^{2}$, which contained 9 scaffolds with dimensions $555 \times 555$ $\times 50 \mu \mathrm{m}^{3}$, for a period of 7 days. Fig. S4† shows the optical microscopy images of the cells cultured on the 3D scaffolds at different time points. In order to visualize cell adhesion on the 3D scaffolds, immunocytochemistry, and the actin/DAPI assay, was employed. However, the high autofluorescence of the photoinitiator presents a great challenge for this assay since it overlaps with the fluorescence of the dyes. In Fig. 2c-f laser scanning confocal microscopy images of the hybrid and the THYMA-hybrid 3D scaffolds with DPSCs, after 7 days of culture, are presented, indicating a good cytoskeleton morphology. Furthermore, the 3D cell cultures were characterized by SEM. Fig. 3 shows the adhesion and growth of DPSCs onto the 3D hybrid and THYMA-hybrid material scaffolds, after 4 

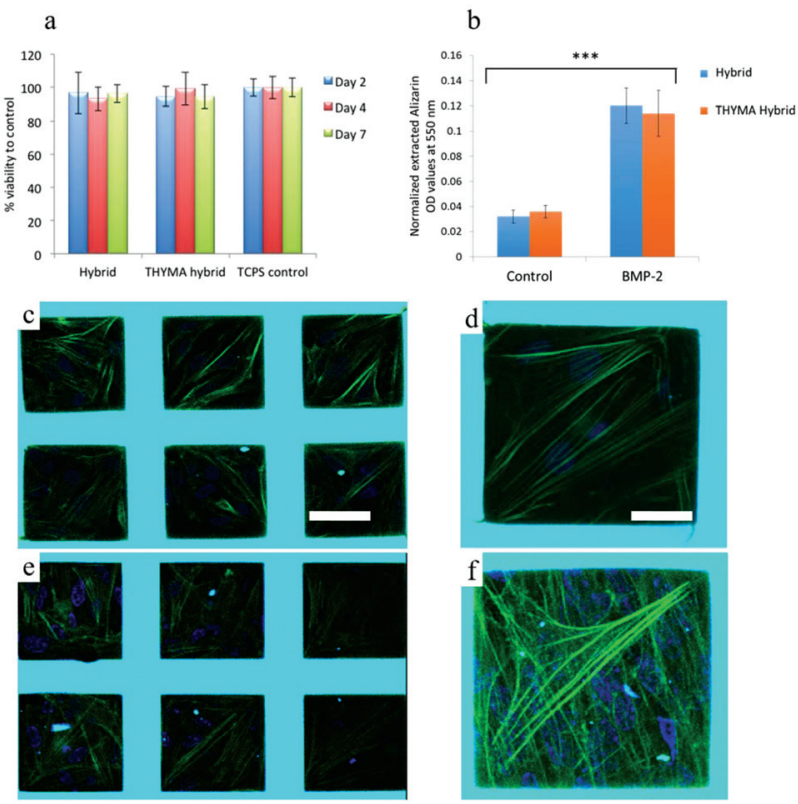

Fig. 2 (a) Viability of DPSCs on the hybrid material films, THYMA-hybrid material films, and the TCPS control; (b) calcium mineralization on the BMP-2-functionalized hybrid and THYMA-hybrid material 3D scaffolds and their respective control scaffolds without BMP-2 for 21 days. (***) designate statistically significant differences $(p=0.0001)$; $(c-f)$ laser scanning confocal microscopy images showing the adhesion of DPSCs on the multi-photon polymerized 3D scaffolds after 7 days in culture. The upper panel images ( $c$ and d) represent stained cells on the hybrid material 3D structures, and the lower panel (e and f) the cells on the THYMA-hybrid material 3D scaffolds. The cells were stained with phalloidin (green: actin filaments) and DAPI (blue: nucleus). Scale bar represents $50 \mu \mathrm{m}$ for images $\mathrm{c}$ and $\mathrm{e}$, and $25 \mu \mathrm{m}$ for (d) and (f).
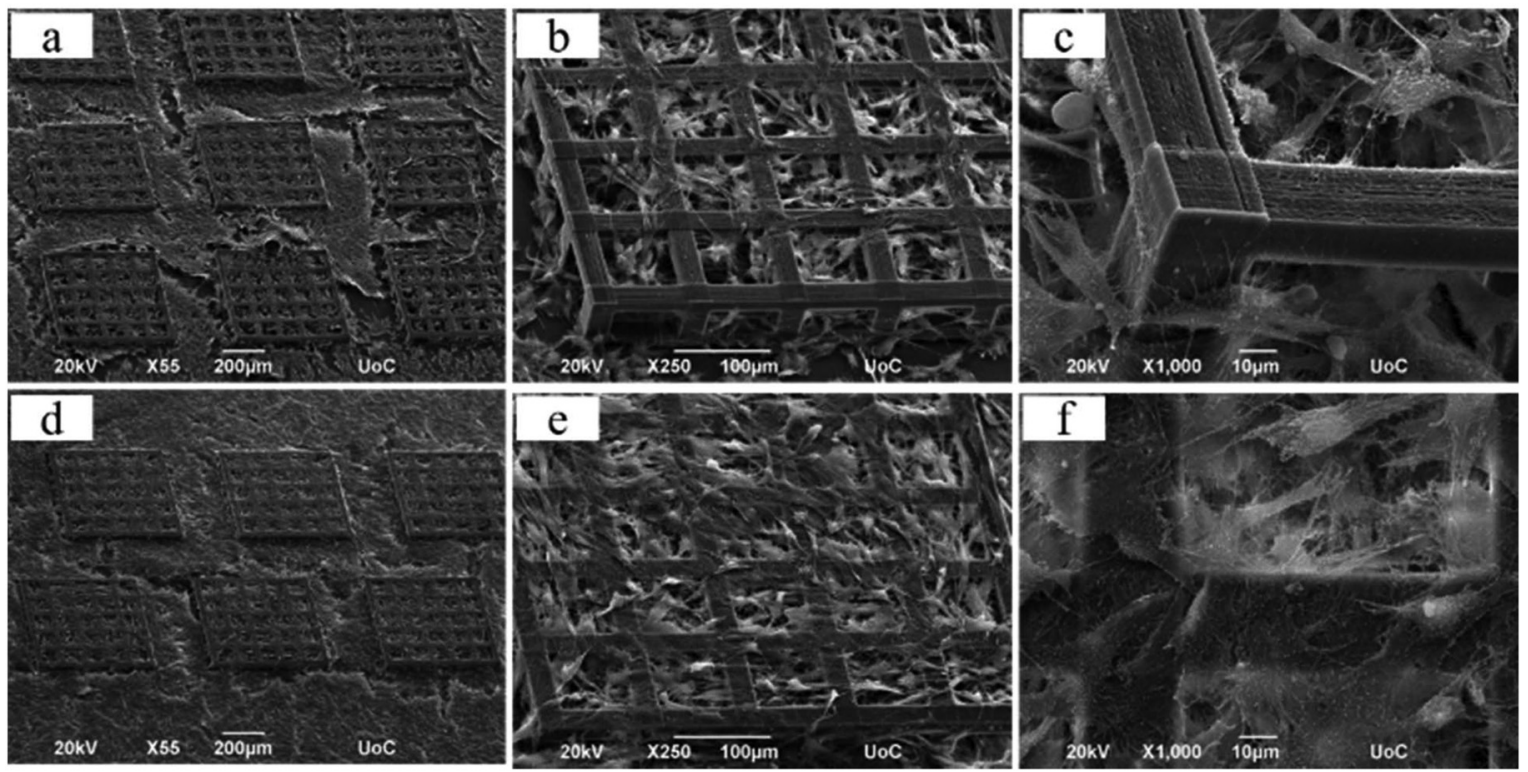

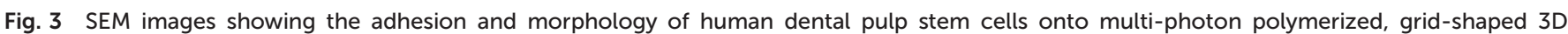

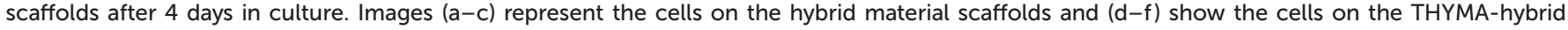
material scaffolds. 

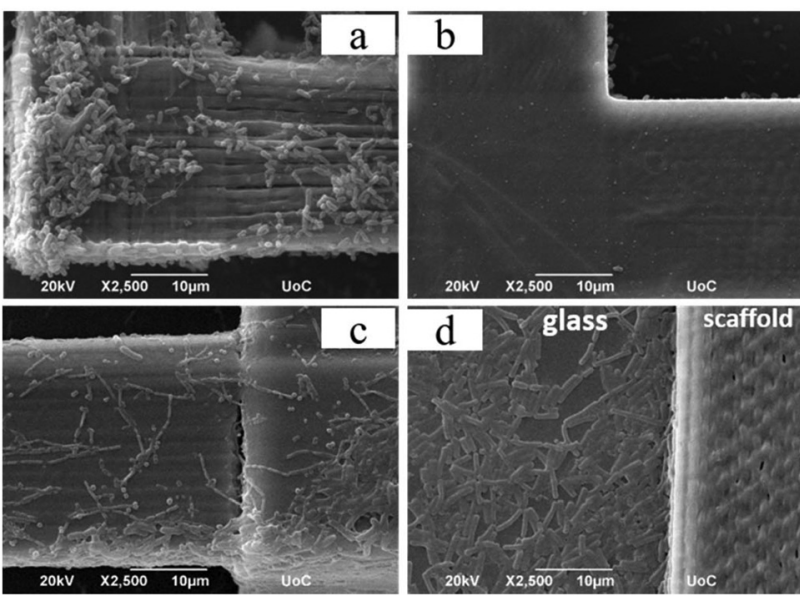

Fig. 4 SEM images of E. coli ( $a$ and b) and B. cereus (c and d) after 4 days in culture. Images (a), (c) represent bacteria cultured on the hybrid material 3D scaffolds, while (b), (d) bacteria cultured on the THYMA-hybrid material 3D scaffolds.

was observed after 1 day of incubation, while bacterial attachment was developed on the surrounding glass substrate (Fig. S6a and $\mathrm{b}, \dagger$ see red arrows). In contrast, on day 4, extensive bacteria growth was found on the hybrid material 3D scaffolds (Fig. 4a), while, no bacteria attachment was observed on the THYMA-hybrid 3D structures (Fig. 4b), verifying the strong antimicrobial action of the latter material. A similar behaviour was found for B. cereus, with the hybrid scaffolds allowing bacterial attachment on the 3D structures on both days 1 and 4 (Fig. S6c $\dagger$ and 4c), while no bacteria were visible on the THYMA-hybrid 3D scaffolds for either time point (Fig. S6 $\dagger \dagger$ and 4d), despite the extensive bacteria growth on the surrounding glass substrate. It is noted, that the difference in the adhesion of the two bacteria strains on the hybrid material scaffolds in day 1 , is attributed to the different physicochemical characteristics of the microorganisms, which has been reported to influence bacteria adherence on a given material. ${ }^{38}$ More importantly, the absence of any bacteria attachment for both $E$. coli and B. cereus on the THYMA-hybrid $3 \mathrm{D}$ scaffolds, after a long incubation period of 4 days, demonstrates the strong antibacterial potency of this material, in contrast to the control hybrid material scaffolds which promote bacteria attachment and growth. This profound antimicrobial action of the THYMA-hybrid 3D scaffolds, against both Gramnegative and Gram-positive bacterial strains, is attributed to the incorporation of the THYMA moieties in the biocompatible hybrid material. The antibacterial mechanism of action of thymol in the THYMA-hybrid 3D scaffolds is attributed to a strong antifouling activity (resistance to bio-adhesion), induced by the increase in the hydrophobicity of the material surface in the presence of THYMA. ${ }^{25}$ This was verified by water contact angle (WCA) measurements on the polymerized hybrid and THYMA-hybrid films, since WCA measurements on the 3D scaffolds are prohibited by their small size and high porosity. A WCA of $58^{\circ}$ was measured on the hybrid material surface, whereas the respective value on the THYMA-hybrid film was $80^{\circ}$ (Fig. S7 $\dagger$ ) verifying the increase in the hydrophobicity of the surface upon the incorporation of THYMA, and therefore the antifouling action of the THYMA-hybrid material.

In conclusion, dual-functional, 3D scaffolds exhibiting simultaneously, biocompatibility and osteogenic potential, as well as an effective antimicrobial activity, were fabricated using MPP. The biocompatibility is based on the hybrid, organic-inorganic, nature of the material, comprising an organicallyfunctionalized $\mathrm{Zr}-\mathrm{Si}$ oxide double network, while the antimicrobial action is due to the presence of a natural essential oil, thymol, which was appropriately modified and covalently bound onto the hybrid material. DPSCs cultured on the $3 \mathrm{D}$ scaffolds proved the material cytocompatibility, evidenced as a strong cell adhesion and proliferation increase on the THYMAhybrid material scaffolds. Additionally, the functionalization of the scaffolds with BMP-2 demonstrated a significant increase of the produced calcium in the matrix, supporting their osteogenic potential. Finally, the THYMA-hybrid material exhibited a strong antimicrobial action against both Gramnegative and Gram-positive bacteria, rendering it very promising for bone, dental and craniofacial tissue engineering applications.

\section{Conflicts of interest}

There are no conflicts to declare.

\section{Acknowledgements}

The authors acknowledge Aleka Manousaki and Stefanos Papadakis for technical assistance with the SEM characterization, Dr Margarita Stapountzi for technical assistance with the laser scanning confocal microscopy and Dr Marios Kidonakis for fruitful discussions. Prof. Athina Bakopoulou from the School of Dentistry, Aristotle University of Thessaloniki, is acknowledged for the generous donation of the human dental pulp stem cells.

\section{References}

1 G. Pasparakis and M. Vamvakaki, Polym. Chem., 2011, 2, 1234-1248.

2 T. Manouras and M. Vamvakaki, Polym. Chem., 2017, 8, 7496.

3 F. Causa, P. A. Netti and L. Ambrosio, Biomaterials, 2007, 28, 5093-5099.

4 Y. C. Li, Y. S. Zhang, A. Akpek, S. R. Shin and A. Khademhosseini, Biofabrication, 2017, 9, 012001.

5 J. Zhang and P. Xiao, Polym. Chem., 2018, 9, 1530-1540.

6 Z. J. Yang, F. F. Wang, C. Zhang, J. Li, R. C. Zhang, Q. Wu, T. H. Chen and P. C. Sun, Polym. Chem., 2019, 10, 33623370 . 
7 N. A. Peppas, J. Z. Hilt, A. Khademhosseini and R. Langer, Adv. Mater., 2006, 18, 1345-1360.

8 K. Parkatzidis, E. Kabouraki, A. Selimis, M. Kaliva, A. Ranella, M. Farsari and M. Vamvakaki, Macromol. Mater. Eng., 2018, 303, 1800458.

9 M. Chatzinikolaidou, T. K. Lichtinger, R. T. Muller and H. P. Jennissen, Acta Biomater., 2010, 6, 4405-4421.

10 M. Malinauskas, P. Danilevicius, D. Baltriukiene, M. Rutkauskas, A. Zukauskas, Z. Kairyte, G. Bickauskaite, V. Purlys, D. Paipulas, V. Bukelskiene and R. Gadonas, Lith. J. Phys., 2010, 50, 75-82.

11 I. Sondi and B. Salopek-Sondi, J. Colloid Interface Sci., 2004, 275, 177-182.

12 J. F. Hernandez-Sierra, F. Ruiz, D. C. C. Pena, F. MartinezGutierrez, A. E. Martinez, A. D. P. Guillen, H. Tapia-Perez and G. A. Martinez-Castanon, Nanomed. Nanotechnol., 2008, 4, 237-240.

13 E. R. Hook, O. J. Owen, C. A. Bellis, J. A. Holder, D. J. O'Sullivan and M. E. Barbour, J. Nanobiotechnol., 2014, 12, 3.

14 Y. Xue, H. N. Xiao and Y. Zhang, Int. J. Mol. Sci., 2015, 16, 3626-3655.

15 L. J. Zhang and R. L. Gallo, Curr. Biol., 2016, 26, R14-R19.

16 S. M. Barbon, N. P. Truong, A. G. Elliott, M. A. Cooper, T. P. Davis, M. R. Whittaker, C. J. Hawker and A. Anastasaki, Polym. Chem., 2020, 11, 84-90.

17 J. L. Grace, J. X. Huang, S.-E. Cheah, N. P. Truong, M. A. Cooper, J. Li, T. P. Davis, J. F. Quinn, T. Velkov and M. R. Whittaker, RSC Adv. , 2016, 6, 15469-15477.

18 M. Pérez-Recalde, I. E. R. Arias and É. B. Hermida, Phytomedicine, 2018, 38, 57-65.

19 M. Wang and T. Tang, J. Orthop.Transl., 2019, 17, 42-54.

20 R. Gyawali and S. A. Ibrahim, Food Control, 2014, 46, 412-429.

21 A. Guarda, J. F. Rubilar, J. Miltz and M. J. Galotto, Int. J. Food Microbiol., 2011, 146, 144-150.

22 M. C. Rota, A. Herrera, R. M. Martinez, J. A. Sotomayor and M. J. Jordan, Food Control, 2008, 19, 681-687.

23 N. Moszner, U. Salz and V. Rheinberger, Polym. Bull., 1994, 33, 7-12.

24 A. Prudencio, A. L. Carbone, J. Griffin and K. E. Uhrich, Macromol. Rapid. Commun., 2009, 30, 1101-1108.
25 S. Bedel, B. Lepoittevin, L. Costa, O. Leroy, D. Dragoe, J. Bruzaud, J. M. Herry, M. Guilbaud, M. N. BellonFontaine and P. Roger, J. Polym. Sci., Part A: Polym. Chem., 2015, 53, 1975-1985.

26 Q. L. Loh and C. Choong, Tissue Eng., Part B, 2013, 19, 485502.

27 M. Farsari and B. N. Chichkov, Nat. Photonics, 2009, 3, 450452.

28 H.-B. Sun and S. Kawata, in NMR 3D Analysis Photopolymerization, Springer, 2004, pp. 169-273.

29 A. Selimis, V. Mironov and M. Farsari, Microelectron. Eng., 2015, 132, 83-89.

30 I. Sakellari, E. Kabouraki, D. Gray, V. Purlys, C. Fotakis, A. Pikulin, N. Bityurin, M. Vamvakaki and M. Farsari, ACS Nano, 2012, 6, 2302-2311.

31 A. Ovsianikov, J. Viertl, B. Chichkov, M. Oubaha, B. MacCraith, I. Sakellari, A. Giakoumaki, D. Gray, M. Vamvakaki, M. Farsari and C. Fotakis, ACS Nano, 2008, 2, 2257-2262.

32 A. Georgopoulou, F. Papadogiannis, A. Batsali, J. Marakis, K. Alpantaki, A. G. Eliopoulos, C. Pontikoglou and M. Chatzinikolaidou, J. Mater. Sci. Mater. Med., 2018, 29, 59.

33 P. Danilevicius, L. Georgiadi, C. J. Pateman, F. Claeyssens, M. Chatzinikolaidou and M. Farsari, Appl. Surf. Sci., 2015, 336, 2-10.

34 A. Bakopoulou, A. Georgopoulou, I. Grivas, C. Bekiari, O. Prymak, K. Loza, M. Epple, G. C. Papadopoulos, P. Koidis and M. Chatzinikolaidou, Dent. Mater., 2019, 35, 310-327.

35 K. Parkatzidis, M. Chatzinikolaidou, M. Kaliva, A. Bakopoulou, M. Farsari and M. Vamvakaki, ACS Biomater. Sci. Eng., 2019, 5, 6161-6170.

36 K. Terzaki, E. Kalloudi, E. Mossou, E. P. Mitchell, V. T. Forsyth, E. Rosseeva, P. Simon, M. Vamvakaki, M. Chatzinikolaidou and A. Mitraki, Biofabrication, 2013, 5, 045002.

37 X. Ge, Z. Li, S. Jing, Y. Wang, N. Li, J. Lu and J. Yu, J. Cell Physiol., 2020, 235, 1209-1221.

38 M. Katsikogianni and Y. Missirlis, Eur. Cells Mater., 2004, 8, 37-57. 